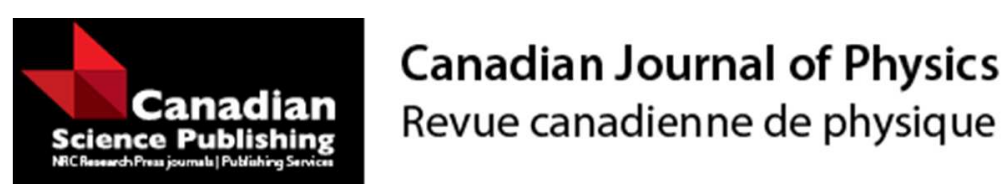

\title{
Formation of non-excited and excited hydrogen in proton lithium inelastic scattering
}

\begin{tabular}{|r|l|}
\hline Journal: & Canadian Journal of Physics \\
\hline Manuscript ID & cjp-2015-0320.R2 \\
\hline Manuscript Type: & Article \\
\hline Date Submitted by the Author: & 19-Aug-2015 \\
\hline Complete List of Authors: & $\begin{array}{l}\text { Elkilany, S.A.; Faculty of Science for girls, Ad Dammam University, ; } \\
\text { Faculty of Science - Kafrelsheikh University, Mathematics } \\
\text { Al-Dhawi, A.A.; University of Dammam, Mathematics Department }\end{array}$ \\
\hline Keyword: & $\begin{array}{l}\text { Inelastic scattering, proton, lithium, hydrogen formation, excited hydrogen } \\
\text { formation }\end{array}$ \\
\hline &
\end{tabular}




\title{
Formation of non-excited and excited hydrogen in proton-lithium inelastic scattering
}

\author{
S A Elkilany and A A Al-Dhawi \\ Department of Mathematics, Faculty of Science, University of Dammam, Kingdom of Saudi Arabia.
}

\begin{abstract}
The collisions of proton with lithium atom are treated for the first time as a three channels problem under the assumption that the elastic and hydrogen formation in non-excited, $H(1 s)$, and excited, $H(2 s)$ ,channels are open. The effect of polarization potentials of the target and hydrogen formation is considered. A one-valence-electron model for the target, based on the Clementi-Roetti Slater-type basis functions, as well as a modified coupled-static approximation are used for calculating the partial and total cross sections of seven partial waves $(0 \leq \ell \leq 6$, where $\ell$ is the total angular momentum) at incident energies between 50 and 500 $\mathrm{keV}$. Our values of the total cross section are in good agreement with previous results.
\end{abstract}

PACSNos.: :: 34.80.Dp; 34.80.Gs; 34.80.Ht

Keywords: Inelastic scattering, proton-lithium, hydrogen formation, excited hydrogen formation

\section{Introduction}

In a recent paper [1] (to be referred to as I), we established a compact technique for treating the collisions of proton with alkali atoms as a three channels problem on the basis of the one-valence-electron model of the targets and the modified coupled-static approximation of the scattering processes. We determined the full forms of the core and static potentials by employing wavefunctions obtained by Clementi and Roetti [2] using a Roothaan-Hatree-Fock approach. This method is a modification of the two channels problem used in the effect of polarization in proton-lithium inelastic scattering [3]. Although the solutions of the scattering problems were carried out using a rather complicated method (in comparison with the first Born and the distorted wave approximations), in which the Green operators adhering to the corresponding Lippmann-Schwinger equations were expressed using partial wave expansions and iterative numerical treatment was 
employed, the compactness and efficiency of the method enable us to investigate collisions of proton with lithium atom.In this case, we have 3 electrons described by the configuration $K(2) 2 s$. Thus, we have orbitals of the type: $1 s^{2}, 2 s$. The Clementi-Roetti wavefunction of each s-orbital is composed of 6 components of Slater-type basis functions (see Ref. [2] ). The effect of polarization potentials of the target and hydrogen formation $H(1 s)$ and $H(2 s)$ is considered.

\section{Theoretical Formalism}

The three-channels scattering problem under investigation can be described by:

$$
p+\operatorname{Li}(2 s)=\left[\begin{array}{lc}
p+\operatorname{Li}(2 s) & \text { Elastic channel (first channel) } \\
H(1 s)+\mathrm{Li}^{+} & H(1 s) \text { formation channel (sec ond channel) } \\
H(2 s)+\mathrm{Li}^{+} & H(2 s) \text { formation channel (third channel) }
\end{array}\right.
$$

The coupled static approximation states that the solution of the three channels scattering problem under consideration is subjected to the following conditions [1]:

$$
\left.\begin{array}{l}
<\Phi_{L i(2 s)}|H-E| \Psi>=0, \\
<\Phi_{H(1 s)}|H-E| \Psi>=0, \\
<\Phi_{H(2 s)}|H-E| \Psi>=0,
\end{array}\right]
$$

where $|\Psi\rangle$ is the total wave function describing each scattering process, i.e.

$$
\begin{aligned}
& |\Psi\rangle=\left|\Phi_{L i(2 s)} \psi_{1}\right\rangle+\left|\Phi_{H(1 s)} \psi_{2}\right\rangle+\left|\Phi_{H(2 s)} \psi_{3}\right\rangle \\
& \Phi_{H(1 s)}=\frac{1}{\sqrt{\pi}} \exp (-\rho)
\end{aligned}
$$

and

$$
\Phi_{H(2 s)}=\frac{1}{\sqrt{32 \pi}}\left(2-\rho^{\prime}\right) \exp \left(-\rho^{\prime} / 2\right)
$$


where $\psi_{1}(x)$ is the wave function describing scattered protons in the first channel, $\psi_{2}(\sigma)$ is the scattering wave function of the second channel and $\psi_{3}\left(\sigma^{\prime}\right)$ is the scattering wave function of the third channel, $\Phi_{L i(2 s)}$ is the wave function for the valence electron of the target atom which is calculated using Ref. [2], $\Phi_{H(1 s)}$ is the ground state wave function of hydrogen atom, $H(1 s)$ and $\Phi_{H(2 s)}$ is the first excited state wave function of hydrogen atom, $H(2 s)$. Our results of the total cross section are obtained under the same conditions of [1](Model I) and we improved its value by considering the effect of polarization potentials of the target and hydrogen formation in our calculations $(H(1 s)$ and $H(2 s))($ Model II) (see Appendix).

The partial elastic and hydrogen formation $(H(1 s), H(2 s))$ cross sections are determined (in $\left.\pi a_{0}^{2}\right)$ by [1]:

$\sigma_{i j}^{(\ell, v)}=\frac{4(2 \ell+1)}{k_{1}^{2}}\left|T_{i j}^{v}\right|^{2}, \quad i, j=1,2,3$

where $k_{1}$ is the momentum of the incident protons, $v$ is the number of iterations and $T_{i j}^{v}$ is the elements of the $3 \times 3$ transition matrix $T^{v}$ which is connected with the reactance matrix $R^{v}$ through the relation: $T^{v}=R^{v}(I-\widetilde{i} R)^{-1}$

where $I$ is a $3 \times 3$ unit matrix and $\tilde{i}=\sqrt{-1}$.

Finally, the total cross sections (in $\pi a_{0}^{2}$ units) are expressed (in $v^{\text {th }}$ iteration) by:

$$
\sigma_{i j}^{v}=\sum_{\ell=0}^{\infty} \sigma_{i j}^{(\ell, v)}, \quad i, j=1,2,3, v>0
$$

\section{Results and discussion}

Our calculations are obtained after 50 iterations (i.e. $v=50$ )and integration range IR $=32 a_{0}$ (remember that IR is the range away from the target nucleus to which all radial integrals are calculated using the Simpson expansions). It has been found that these conditions are enough for obtaining excellent convergence in the elements of $R^{v}$ (see Ref. [1]). 
Figure 1 contains the partial and total elastic cross section, $\sigma_{11}$ of Model II, for $0 \leq \ell \leq 6$ that correspond to incident energies between 50 and $250 \mathrm{keV}$. Figure 1 demonstrates the behavior of the partial elastic cross sections at all values of the total angular momentum $\ell$. It also shows the important role played by the S, P and D waves at all considered incident energies. The values of the cross sections decrease steadily with $k_{1}^{2}$ and the seven partial waves employed are quite satisfactory for calculating them (since the higher partial waves are very small). Figure 2 involves the partial and total hydrogen formation cross sections $\sigma_{12}$ of the non-excited hydrogen, $H(1 s)$ of Model II. We also show the partial and total hydrogen formation cross sections $\sigma_{13}$ of excited hydrogen, $H(2 s)$ of Model II in figure 3. Figures 2, 3 show similar behavior of the partial and total cross sections as it is in the previous elastic results. The most interesting results of our investigations are presented in table 1 and figures 4 and 5, where we show a comparison between our values of the total cross section of $H(1 s)$ and $H(2 s)$ (Model I and Model II) with those determined by different authors using different approaches. Columns 2 and 3 in table 1 show the present total hydrogen formation, $H(1 s)$, cross sections $\sigma_{12}$ (Model I and Model II, respectively). Column 4 gives the values of the total hydrogen formation, $H(1 s)$, cross sections $\sigma_{12}$ obtained by Daniele et al. [4] using the eikonal-approximation (eik), developed previously within the framework of time-independent impact parameter method for high-energy proton-alkali atom charge transfer. The next two columns contain the values of the total hydrogen formation, $H(1 s)$, cross sections $\sigma_{12}$ of Ferrante et al. [5] using Oppenheimer, Brinkman and Kramers (OBK) and first Born approximation (FBA) approximations, respectively (see Fig. 4). Present total excited hydrogen formation, $H(2 s)$, cross sections $\sigma_{13}$ (Model I and Model II, respectively) are displayed in columns 7 and 8 . At the last three columns, we show the values of the total excited hydrogen formation, $H(2 s)$, cross sections $\sigma_{13}$ obtained by Ferrante et al. [5] using OBK approximation, Banyard et al. [6] using the continuum distorted wave approximation (CDW), and by Tiwari[7] using the Coulomb projected Born (CPB), respectively (see Fig. 5). Our calculations of the total hydrogen formation, $H(1 s)$, cross sections $\sigma_{12}$ (Model I) show that our values of the present total cross sections are about 14-19\% lower than those of Ref. [5] (FBA) at the interval of the incident energy 
$70-200 \mathrm{keV}$ and are about 7-13\% higher than those of Ref. [5] (FBA) for model II at the same interval of energy (see

Fig. 4). We can also conclude that our values of the total hydrogen formation, $H(1 \mathrm{~s})$, cross sections $\sigma_{12}$ of model II have the same trend and in good agreement with those of the compared results at all considered incident energies. Our results for excited hydrogen formation, $H(2 s)$, cross sections $\sigma_{13}$ of Model I at the interval of the incident energy $50-200 \mathrm{keV}$ are about $10-22 \%$ lower than those of Ref. [7], and are about $0.4-7 \%$ lower than those of Ref. [7] (CPB) for model II on the same interval of energy except at $k_{1}^{2}=150 \mathrm{keV}$ our value is about $5 \%$ higher than the compared result of Ref. [7] (CPB) (see Fig. 5). From the above discussion, it may be stated that our values of the total excited hydrogen function, $H(2 s)$, cross sections $\sigma_{13}$ of model II have the same trend of the compared results and in good agreement with the available results.

\section{Conclusions}

Proton-lithium inelastic scattering is studied for the first time as a three channels problem using the modified coupled static approximation. Our interest is focused on the formation of ground, $H(1 s)$, and excited hydrogen $H(2 s)$. Our results of the total hydrogen formation, $H(1 s)$ and $H(2 s)$ (Model I) are improved by considering the polarization potentials of the target and hydrogen formation atoms, $H(1 s)$ and $H(2 s)$, (Model II). The difference between the three channels problem and the two channels problem is in improving the total cross sections of the considered channel by considering the effect of the two kernels of the other two channels (in two channels problem we have only one kernel) . Our problem shows that three channels coupled static approximation give good agreement with previous results especially if we take the polarization potentials of the target and hydrogen formation atoms in our calculation.

\section{Acknowledgments}

The authors are pleased to acknowledge the financial support of this study by the Scientific Research Deanship of University of Dammam, under Project no. 2015001. 


\section{References}

[ 1] S. A. Elkilany, Can. J. Phys., on line first 27 April 2015.

http://nrcresearchpress.com/doi/abs/10.1139/cjp-2015-0116? src=recsys\&journalCode=cjp\#.VU8PXo6qqkq

[2] E. Clementi and C. Roetti, At. Data Nucl. Data Tables, 14, 177 (1974).

http://lib.gen.in/next/MTAuMTAxNi9zMDA5Mi02NDB4KDc0KTgwMDE2LTE=/clementi1974.pdf

http://www.sciencedirect.com.sci-hub.org/science/article/pii/S0092640X74800161

[3] S. A. Elkilany and A. A. Al-Dawy, Chin. Phys. Lett. 31(9), 093401 (2014).

http://iopscience.iop.org/0256-307X/31/9/093401

[4] R. Daniele, G. Ferrante and E. Fiordilino, IL Nuovo Cimento, 54B(1), 185 (1979).

http://link.springer.com/article/10.1007/BF02908234

[5] G. Ferrante, E. Fiordilino and M. Zarcone, IL Nuovo Cimento, 52B(2), 151 (1979).

http://link.springer.com.sci-hub.org/article/10.1007/BF02739030

[6] K. E. Banyard and G. W. Shirtcliffe, J. Phys. B: at. Mol. Phys. 12(19), 3247 (1979).

http://iopscience.iop.org.sci-hub.org/0022-3700/12/19/010

[7] Y. N. Tiwari, Pramana J. Phys. 70(4), 753 (2008).

http://lib.gen.in/next/MTAuMTAwNy9zMTIwNDMtMDA4LTAwMzYteA==/tiwari2008.pdf

[8] G. Peach, Comments At. Mol. Phys. 11 (3-5), 101 (1982) Private communication.

[9] M. A. Abdel-Raouf, Acta Physica Hungarica, 63(1-2), 21 (1988).

http://link.springer.com/article/10.1007\%2FBF03155754 
Table 1. Present total non excited hydrogen formation, $H(1 s)$ cross sections ( $\sigma_{12}$ in $\pi a_{0}^{2}$ ) and total excited hydrogen formation, $H(2 s)$ cross sections ( $\sigma_{13}$ in $\pi a_{0}^{2}$ ) of $p-L i$ scattering (Model I and Model II) are compared against theoretical results ([4]- [7]).

\begin{tabular}{|c|c|c|c|c|c|c|c|c|c|c|}
\hline $\begin{array}{c}\mathrm{k}^{2} \\
(\mathrm{keV})\end{array}$ & $\begin{array}{c}\text { Present } \\
\sigma_{12} \\
\text { Model I }\end{array}$ & $\begin{array}{c}\text { Present } \\
\sigma_{12} \\
\text { Model II }\end{array}$ & $\begin{array}{c}\text { Ref. [4] } \\
\sigma_{12} \\
(\text { eik) }\end{array}$ & $\begin{array}{c}\text { Ref. [5] } \\
\sigma_{12} \\
(\mathrm{OBK}) \\
\end{array}$ & $\begin{array}{c}\text { Ref. [5] } \\
\sigma_{12} \\
\text { (FBA) }\end{array}$ & $\begin{array}{c}\text { Present } \\
\sigma_{13} \\
\text { Model I }\end{array}$ & $\begin{array}{c}\text { Present } \\
\sigma_{13} \\
\text { Model II } \\
\end{array}$ & $\begin{array}{c}\text { Ref. [5] } \\
\sigma_{13} \\
(\mathrm{OBK})\end{array}$ & $\begin{array}{c}\text { Ref. [6] } \\
\sigma_{13} \\
(\mathrm{CDW}) \\
\end{array}$ & $\begin{array}{c}\text { Ref. [7] } \\
\sigma_{13} \\
(\mathrm{CPB}) \\
\end{array}$ \\
\hline 50 & $5.8693 \mathrm{E}-02$ & 7.6673E-02 & & & & $3.0989 \mathrm{E}-03$ & $3.4818 \mathrm{E}-03$ & $6.5 \mathrm{E}-01$ & & $3.596 \mathrm{E}-03$ \\
\hline 60 & $5.5421 \mathrm{E}-02$ & 7.2531E-02 & & & & $2.2312 \mathrm{E}-03$ & $2.4709 \mathrm{E}-03$ & & & \\
\hline 70 & $4.2036 \mathrm{E}-02$ & $5.5215 \mathrm{E}-02$ & $1.1975 \mathrm{E}-02$ & 7.8162E-02 & $5.1665 \mathrm{E}-02$ & $1.6064 \mathrm{E}-03$ & $1.7903 \mathrm{E}-03$ & & & \\
\hline 80 & $1.4598 \mathrm{E}-02$ & $1.9144 \mathrm{E}-02$ & $8.6291 \mathrm{E}-03$ & $2.6978 \mathrm{E}-02$ & $1.7832 \mathrm{E}-02$ & $1.1567 \mathrm{E}-03$ & $1.2988 \mathrm{E}-03$ & & & \\
\hline 90 & $6.1937 \mathrm{E}-03$ & $8.1592 \mathrm{E}-03$ & $6.4477 \mathrm{E}-03$ & $1.1458 \mathrm{E}-02$ & 7.5737E-03 & $8.3280 \mathrm{E}-04$ & 9.3929E-04 & & & \\
\hline 100 & $3.7011 \mathrm{E}-03$ & 4.8892E-03 & 4.8947E-03 & $6.8400 \mathrm{E}-03$ & $4.5212 \mathrm{E}-03$ & 5.9961E-04 & $6.7204 \mathrm{E}-04$ & $1.0 \mathrm{E}-03$ & & $6.746 \mathrm{E}-04$ \\
\hline 110 & $2.9396 \mathrm{E}-03$ & $3.9045 \mathrm{E}-03$ & $3.7748 \mathrm{E}-03$ & $5.4443 \mathrm{E}-03$ & $3.5986 \mathrm{E}-03$ & $4.3172 \mathrm{E}-04$ & 4.8867E-04 & & & \\
\hline 120 & $2.6691 \mathrm{E}-03$ & $3.5310 \mathrm{E}-03$ & $2.9358 \mathrm{E}-03$ & $4.8988 \mathrm{E}-03$ & $3.2381 \mathrm{E}-03$ & $3.1084 \mathrm{E}-04$ & $3.5165 \mathrm{E}-04$ & & & \\
\hline 130 & $2.4826 \mathrm{E}-03$ & $3.2753 \mathrm{E}-03$ & & - & & $2.2380 \mathrm{E}-04$ & $2.5505 \mathrm{E}-04$ & & & \\
\hline 140 & $2.2797 \mathrm{E}-03$ & $2.9965 \mathrm{E}-03$ & $1.8760 \mathrm{E}-03$ & $4.1165 \mathrm{E}-03$ & $2.7210 \mathrm{E}-03$ & $1.6114 \mathrm{E}-04$ & $1.8534 \mathrm{E}-04$ & & & \\
\hline 150 & $2.0605 \mathrm{E}-03$ & $2.7020 \mathrm{E}-03$ & & ) & & $1.1602 \mathrm{E}-04$ & $1.3532 \mathrm{E}-04$ & $4.9 \mathrm{E}-03$ & & $1.294 \mathrm{E}-04$ \\
\hline 160 & $1.8422 \mathrm{E}-03$ & 2.4092E-03 & $1.2191 \mathrm{E}-03$ & $3.2779 \mathrm{E}-03$ & $2.1667 \mathrm{E}-03$ & 8.3534E-05 & $9.6048 \mathrm{E}-05$ & & & \\
\hline 170 & $1.6397 \mathrm{E}-03$ & $2.1258 \mathrm{E}-03$ & & & & $6.0145 \mathrm{E}-05$ & $6.9608 \mathrm{E}-05$ & & & \\
\hline 180 & $1.4211 \mathrm{E}-03$ & $1.8564 \mathrm{E}-03$ & 8.3367E-04 & $2.5032 \mathrm{E}-03$ & $1.6546 \mathrm{E}-03$ & $4.3304 \mathrm{E}-05$ & $5.0507 \mathrm{E}-05$ & & & \\
\hline 190 & $1.2318 \mathrm{E}-03$ & $1.6122 \mathrm{E}-03$ & & & 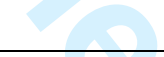 & $3.1179 \mathrm{E}-05$ & 3.6534E-05 & & & \\
\hline 200 & $1.0658 \mathrm{E}-03$ & $1.4000 \mathrm{E}-03$ & 5.6149E-04 & $1.8730 \mathrm{E}-03$ & $1.2380 \mathrm{E}-03$ & 2.5849E-05 & $3.0662 \mathrm{E}-05$ & $2.8 \mathrm{E}-03$ & $1.048 \mathrm{E}-05$ & $3.294 \mathrm{E}-05$ \\
\hline 210 & $5.7259 \mathrm{E}-04$ & 7.5089E-04 & & & & $1.6163 \mathrm{E}-05$ & $1.9014 \mathrm{E}-05$ & & & \\
\hline 220 & $1.2786 \mathrm{E}-04$ & $5.1054 \mathrm{E}-04$ & & & & $1.1638 \mathrm{E}-05$ & $1.3685 \mathrm{E}-05$ & & & \\
\hline 230 & $3.4424 \mathrm{E}-04$ & 4.5474E-04 & & & & $8.3790 \mathrm{E}-06$ & $9.9274 \mathrm{E}-06$ & & & \\
\hline 240 & $2.7079 \mathrm{E}-04$ & 3.5967E-04 & & & & $6.0329 \mathrm{E}-06$ & 7.2153E-06 & & & \\
\hline 250 & $2.4867 \mathrm{E}-04$ & $3.2898 \mathrm{E}-04$ & & & & 4.3437E-06 & $5.2692 \mathrm{E}-06$ & & & $1.327 \mathrm{E}-05$ \\
\hline 300 & $2.3485 \mathrm{E}-04$ & $3.0984 \mathrm{E}-04$ & & & & $2.9857 \mathrm{E}-06$ & $3.2451 \mathrm{E}-06$ & & & $8.489 \mathrm{E}-06$ \\
\hline 350 & $9.4802 \mathrm{E}-06$ & $1.2461 \mathrm{E}-05$ & & & & $2.0685 \mathrm{E}-06$ & $3.0358 \mathrm{E}-06$ & & & \\
\hline 400 & $6.4581 \mathrm{E}-06$ & $8.4688 \mathrm{E}-06$ & & & & $1.4869 \mathrm{E}-06$ & $2.1945 \mathrm{E}-06$ & & & \\
\hline 450 & 4.1927E-06 & $5.4832 \mathrm{E}-06$ & & & & $1.1150 \mathrm{E}-06$ & $1.2234 \mathrm{E}-06$ & & & \\
\hline 500 & $1.4188 \mathrm{E}-06$ & $1.8393 \mathrm{E}-06$ & & & & $8.0153 \mathrm{E}-07$ & $9.7696 \mathrm{E}-07$ & & $5.212 \mathrm{E}-07$ & $9.828 \mathrm{E}-07$ \\
\hline
\end{tabular}




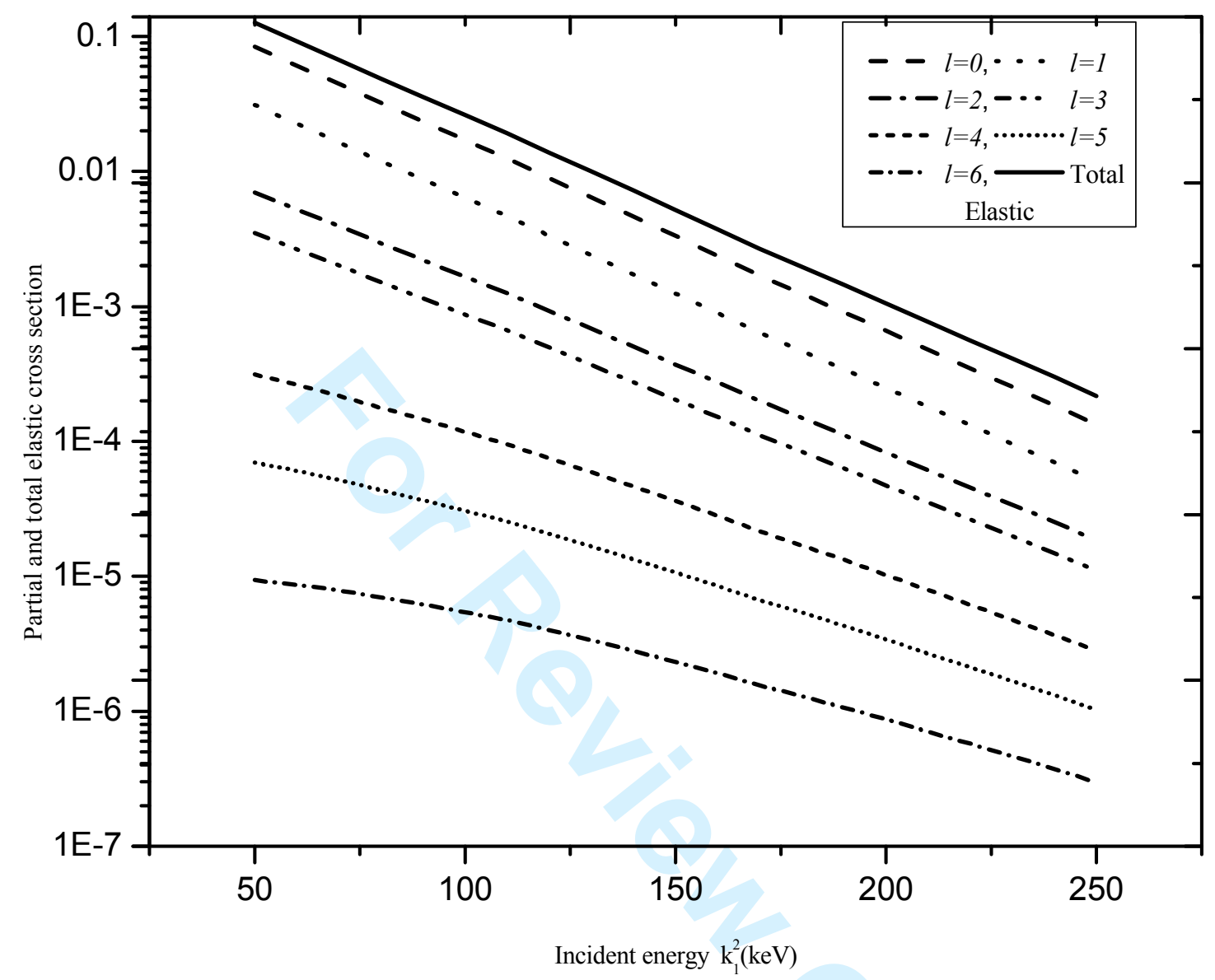

Fig. 1 Present partial and total elastic cross sections (in $\pi a_{0}^{2}$ ) of $p-L i$ scattering (Model II). 


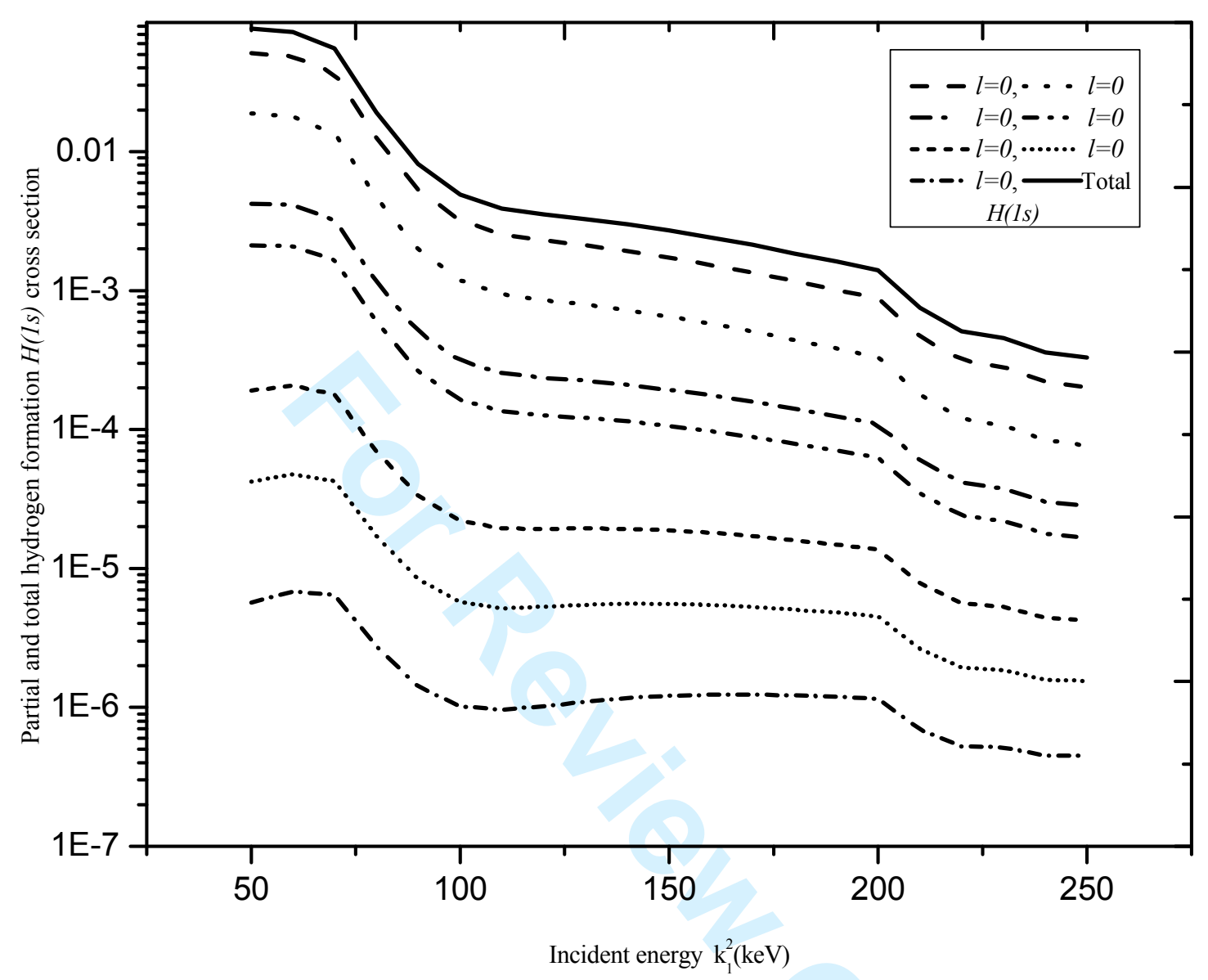

Fig. 2 Present partial and total $H(1 s)$ formation cross sections (in $\pi a_{0}^{2}$ ) of $p$ - Li scattering (Model II) . 


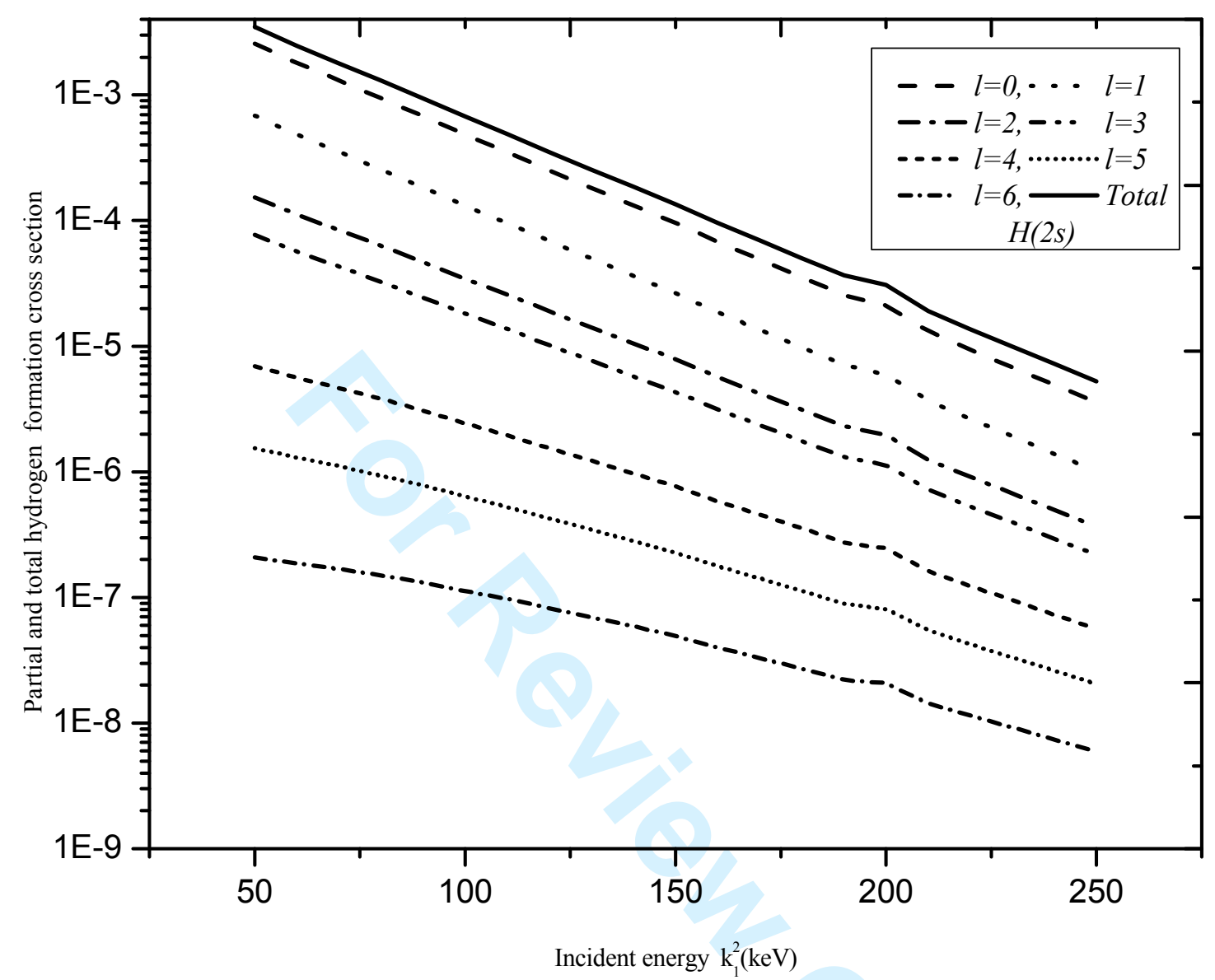

Fig. 3 Present partial and total $H(2 s)$ formation cross sections (in $\pi a_{0}^{2}$ ) of $p$-Li scattering (Model II). 


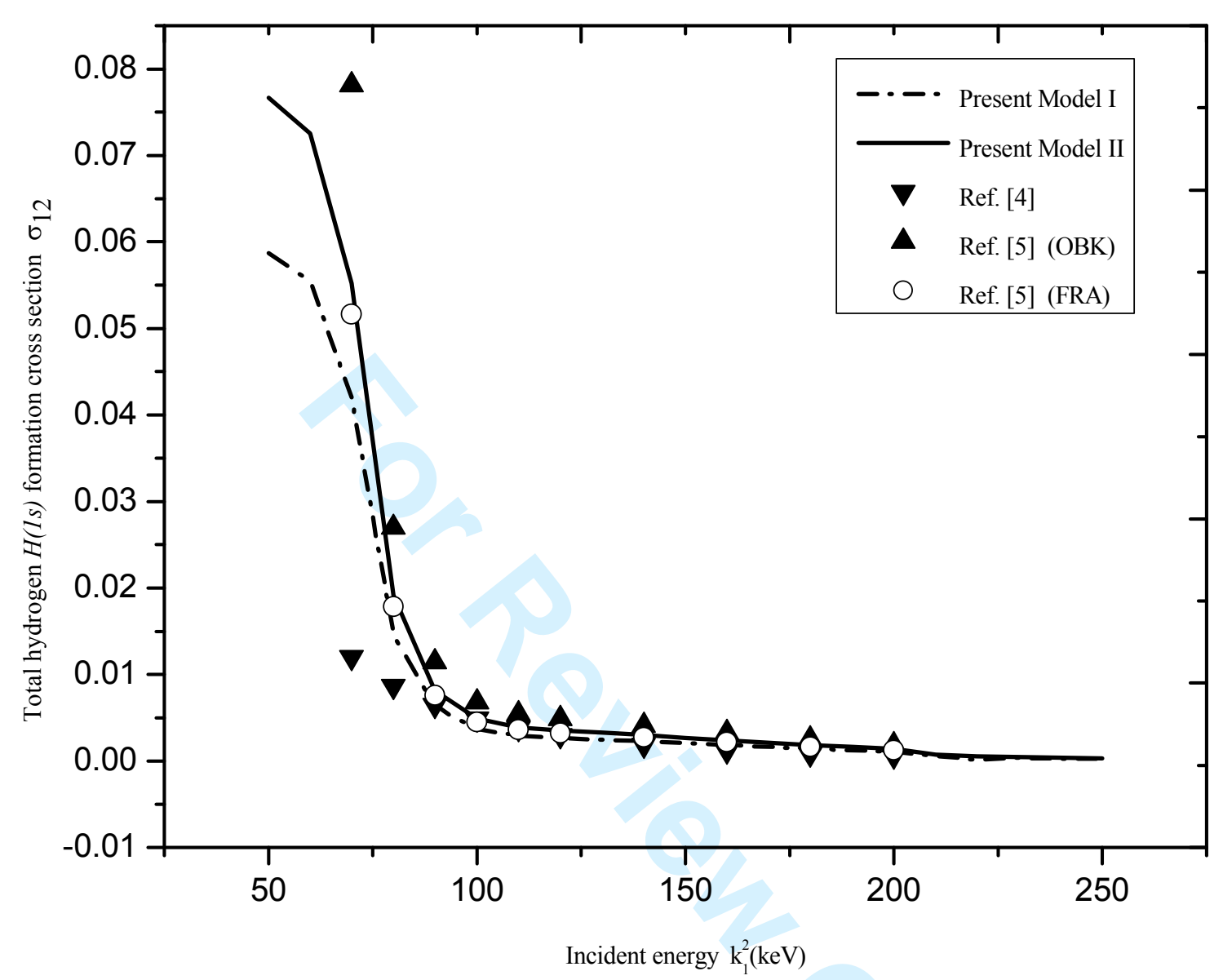

Fig. 4 Present total $H(1 s)$ formation cross sections $\left(\sigma_{12}\right.$ in $\left.\pi a_{0}^{2}\right)$ of $p-L i$ scattering are compared against theoretical results ([4] and [5]). 


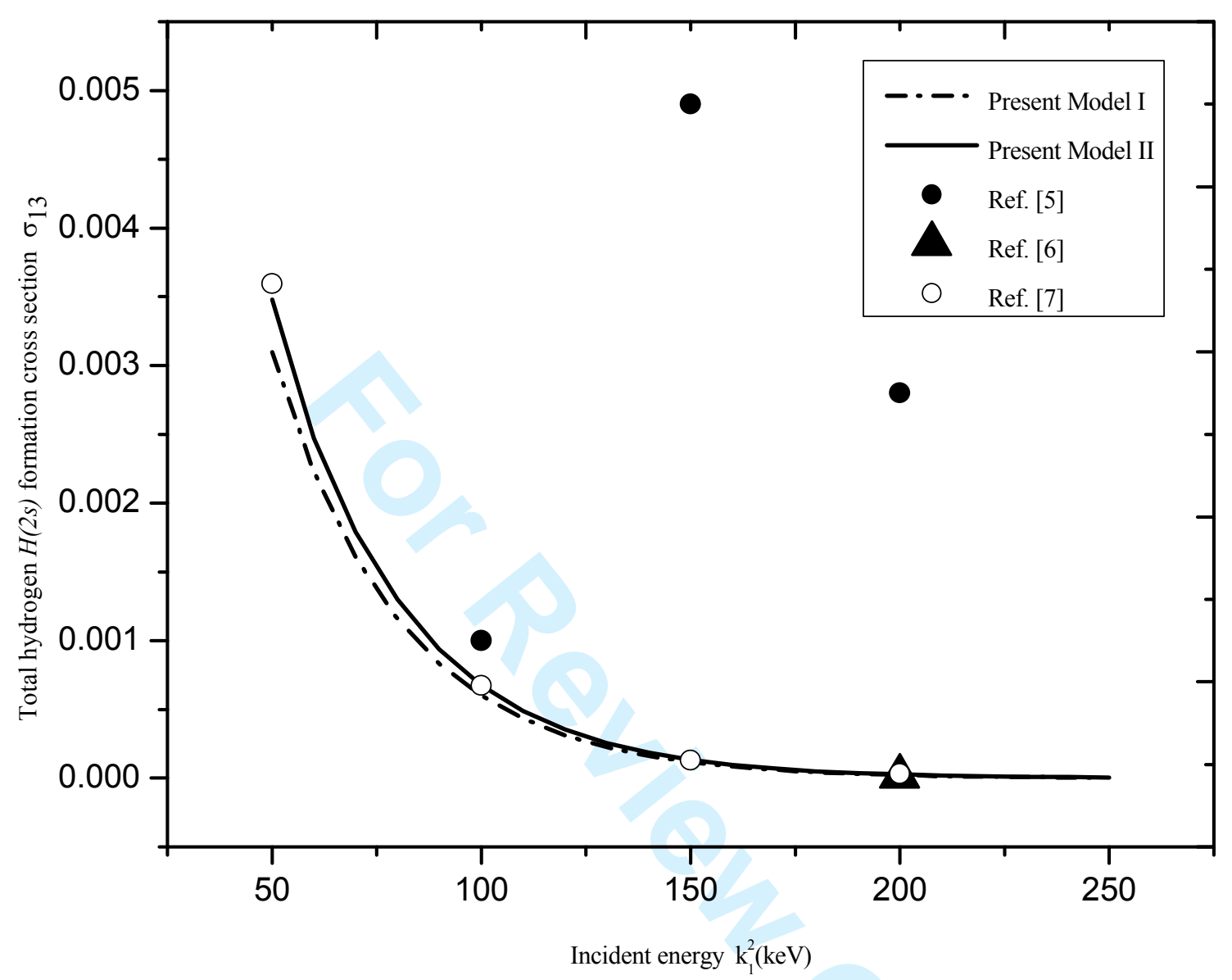

Fig. 5 Present total $H(2 s)$ formation cross sections $\left(\sigma_{13}\right.$ in $\left.\pi a_{0}^{2}\right)$ of $p-L i$ scattering are compared against theoretical results ([5], [6] and [7]). 


\section{Appendix}

The potentials $U_{s t}^{(1)}(x), U_{s t}^{(2)}(\sigma)$ and $U_{s t}^{(3)}\left(\sigma^{\prime}\right)$ are defined by [1]:

$U_{s t}^{(1)}(x)=<\Phi_{L i(2 s)}(r)\left|V_{\text {int }}^{(1)}\right| \Phi_{L i(2 s)}(r)>$

$U_{s t}^{(2)}(\sigma)=<\Phi_{H(1 s)}(\rho)\left|V_{\text {int }}^{(2)}\right| \Phi_{H(1 s)}(\rho)>$

and

$U_{s t}^{(3)}\left(\sigma^{\prime}\right)=<\Phi_{H(2 s)}\left(\rho^{\prime}\right)\left|V_{\text {int }}^{(3)}\right| \Phi_{H(2 s)}\left(\rho^{\prime}\right)>$

where $V_{\text {int }}^{(1)}, V_{\text {int }}^{(2)}$ and $V_{\text {int }}^{(3)}$ are the interaction between the particles of the three channels, respectively.

The effect of the polarization potentials of $L i(2 s), H(1 s)$ and $H(2 s)$ atoms i.e. $V_{p o l}^{L i(2 s)}(x), V_{p o l}^{H(1 s)}(\sigma)$ and $V_{p o l}^{H(2 s)}\left(\sigma^{\prime}\right)$ can be carried out by replacing $U_{s t}^{(1)}(x), U_{s t}^{(2)}(\sigma)$ and $U_{s t}^{(3)}\left(\sigma^{\prime}\right)$ by $U^{(1)}(x), U^{(2)}(\sigma)$ and $U^{(3)}\left(\sigma^{\prime}\right)$, respectively, such that

$U^{(1)}(x)=U_{s t}^{(1)}(x)+V_{p o l}^{L i(2 s)}(x)$,

$U^{(2)}(\sigma)=U_{s t}^{(2)}(\sigma)+V_{p o l}^{H(1 s)}(\sigma)$

and

$U^{(3)}\left(\sigma^{\prime}\right)=U_{s t}^{(3)}\left(\sigma^{\prime}\right)+V_{p o l}^{H(2 s)}\left(\sigma^{\prime}\right)$

We choose the polarization potential of the first channel, $V_{p o l}^{L i(2 s)}(x)$, in Peach form [8]

$V_{p o l}^{L i(2 s)}(x)=-\frac{\alpha}{x^{4}}\left\{1.0-\left(1.0+\gamma x+\frac{(\gamma x)^{2}}{2}\right) e^{-\gamma x}\right\}$

where $\alpha=0.19$ and $\gamma=3.91$

The polarization potentials $V_{p o l}^{H(1 s)}(\sigma)$ of channel 2 and $V_{p o l}^{H(2 s)}\left(\sigma^{\prime}\right)$ of channel 3 are added to $U_{s t}^{(2)}(\sigma)$ and $U_{s t}^{(3)}\left(\sigma^{\prime}\right)$, respectively, while the coupled static picture of the system is considered. $V_{p o l}^{H(1 s)}(\sigma)$, which is added to include all p-states of $n=2$, i.e. $H(1 s+2 p)$ is defined by [9]. 
Let

$$
\Phi_{H(n p)}(\rho)=\left(\frac{8}{43}\right)^{\frac{1}{2}} \rho\left(1+\frac{\rho}{2}\right) e^{-\rho}
$$

$$
V^{1 s}(\sigma)=\left\langle\Phi_{H(1 s)}(\rho)\left|V_{\text {int }}^{(1)}\right| \Phi_{H(n p)}(\rho)\right\rangle
$$

and

$$
U^{1 s}(\sigma)=\left\langle\Phi_{H(n p)}(\rho)\left|V_{\text {int }}^{(1)}\right| \Phi_{H(n p)}(\rho)\right\rangle
$$

then

$$
V_{p o l}^{H(1 s)}(\sigma)=\beta^{1 s}(\sigma) V^{1 s}(\sigma)
$$

The function $\beta^{1 s}(\sigma)$ is calculated as follows: let $\Delta E^{1 s}=E_{\mathrm{H}(1 \mathrm{~s})}-E_{H^{*}}$, (where $E_{H(1 s)}=-1.0$ Ryd is binding energy of $H(1 s), E_{H^{*}}=-21 / 129 R y d$ is the binding energy of the polarized hydrogen, and $w^{1 s}=\Delta E^{1 s} / V^{1 s}(\sigma)$. Also, we consider the two functions $\beta^{1 s \pm}$ such that $\beta^{1 s \pm}(\sigma)=\frac{1}{2}\left\{-w^{1 s} \pm\left(\left(w^{1 s}\right)^{2}+4\right)^{\frac{1}{2}}\right\}$

The adiabatic energy of $H(1 s)$ within the field of a unit positive charge is found to be

$$
E_{a d}^{H(1 s)}=\left\{E_{H(1 s)}+U_{s t}^{(1)}+2\left(\beta^{1 s \pm}\right) V^{1 s}+\left[E_{H^{*}}+U^{1 s}\right]\left(\beta^{1 s \pm}\right)^{2}\right\} /\left[1+\left(\beta^{1 s \pm}\right)^{2}\right]
$$

The correct $\beta$ for calculating $V_{p o l}^{H(1 s)}(\sigma)$ is that one of $\beta^{1 s+}$ and $\beta^{1 s^{-}}$which yields a minimum value for $E_{a d}^{H(1 s)}$.

We, also, have $V_{p o l}^{H(2 s)}\left(\sigma^{\prime}\right)$ for channel 3 by replacing $1 s$ by $2 s$ in the previous calculation and $E_{H(2 s)}=-0.25 R y d$ which is the first excited 2s-state energy of hydrogen atom. 